\title{
Stochastic Lie bracket (derivation, derivation) in MB-algebras
}

\author{
Masoumeh Madadi ${ }^{1}$, Reza Saadati ${ }^{*}$ D, Choonkil Park ${ }^{3}$ and John Michael Rassias ${ }^{4}$
}

"Correspondence: rsaadati@eml.cc; rsaadati@iust.ac.ir

${ }^{2}$ School of Mathematics, Iran University of Science and

Technology, Tehran, Iran Full list of author information is available at the end of the article

\begin{abstract}
By a stochastic controller, we make stable the pseudo stochastic Lie bracket (derivation, derivation) in complex MB-algebras. Next, we get an approximation by a stochastic Lie bracket (derivation, derivation) and calculate the maximum error of the estimate.
\end{abstract}

MSC: 47B47; 47H10; 39B52; 39B72; 46L57

Keywords: Lie bracket (derivation, derivation); Stability; Stochastic controller; Fixed point technique; Banach algebra; Random operator inequality; Menger space

\section{Introduction}

Let $(\Omega, \mathfrak{T}, \mu)$ be a probability measure space. Assume that $\left(T, \mathfrak{B}_{T}\right)$ is a Borel measureable space, in which $T$ is an MB-space and $G, H: \Omega \times T \rightarrow T$ are random derivations. In MBspaces, first we solve the (additive, additive) $-(\omega, v)$ random operator inequality

$$
\begin{aligned}
& \xi_{\tau}^{G(\gamma, t+s)-G(\gamma, t)-G(\gamma, s)} * \xi_{\tau}^{H(\gamma, t+s)+H(\gamma, t-s)-2 H(\gamma, t)} \\
& \quad \geq \xi_{\tau}^{\omega\left(2 G\left(\gamma, \frac{t+s}{2}\right)-G(\gamma, t)-G(\gamma, s)\right)} * \xi_{\tau}^{\nu\left(2 H\left(\gamma, \frac{t+s}{2}\right)+2 H\left(\gamma, \frac{t-s}{2}\right)-2 H(\gamma, t)\right)},
\end{aligned}
$$

where $\omega, v$ are fixed nonzero complex numbers. By a stochastic controller we make stable the pseudo stochastic Lie bracket (derivation, derivation) in complex MB-algebras, associated to the above (additive, additive) $-(\omega, v)$ random operator inequality and the following random operator inequality:

$$
\xi_{\tau}^{[G, H](\gamma, t s)-[G, H](\gamma, t) s-t[G, H](\gamma, s)} * \xi_{\tau}^{H(\gamma, t s)-H(\gamma, t) s-t H(\gamma, s)} \geq \varphi_{\tau}^{t, s} .
$$

The mentioned process is said to show Hyers-Ulam stability for the (additive, additive)$(\omega, v)$ random operator inequality (1.1).

\section{Preliminaries}

Let $\Xi^{+}$be the set of distribution mappings, i.e., the set of all mappings $\rho: \mathbb{R} \cup\{-\infty, \infty\} \rightarrow$ $[0,1]$, writing $\rho_{\tau}$ for $\rho(\tau)$, such that $\rho$ is left continuous and increasing on $\mathbb{R}$. $O^{+} \subseteq \Xi^{+}$

(c) The Author(s) 2020. This article is licensed under a Creative Commons Attribution 4.0 International License, which permits use, sharing, adaptation, distribution and reproduction in any medium or format, as long as you give appropriate credit to the original author(s) and the source, provide a link to the Creative Commons licence, and indicate if changes were made. The images or other third party material in this article are included in the article's Creative Commons licence, unless indicated otherwise in a credit line to the material. If material is not included in the article's Creative Commons licence and your intended use is not permitted by statutory regulation or exceeds the permitted use, you will need to obtain permission directly from the copyright holder. To view a copy of this licence, visit http://creativecommons.org/licenses/by/4.0/. 
includes all mappings $\rho \in \Xi^{+}$for which $\ell^{-} \rho_{+\infty}$ is one and $\ell^{-} \rho_{\tau}$ is the left limit of the mapping $\rho$ at the point $\tau$, i.e., $\ell^{-} \rho_{\tau}=\lim _{\sigma \rightarrow \tau^{-}} \rho_{\sigma}$.

In $\Xi^{+}$, we define " $\leq$" as follows:

$\rho \leq \varrho \quad$ if and only if $\quad \rho_{\tau} \leq \varrho_{\tau}$

for each $\tau$ in $\mathbb{R}$ (partially ordered). Note that the function $\vartheta^{u}$ defined by

$$
\vartheta_{s}^{u}= \begin{cases}0, & \text { if } s \leq u, \\ 1, & \text { if } s>u,\end{cases}
$$

is an element of $\Xi^{+}$and $\vartheta^{0}$ is the maximal element in this space (for details, see [1-3]).

Definition 2.1 ([1, 4]) Denote by $I$ the interval $[0,1]$. A continuous triangular norm (shortly, a $c t$-norm) is a continuous binary operation $*$ from $I^{2}$ to $I$ such that

(a) $\varsigma * \tau=\tau * \varsigma$ and $\varsigma *(\tau * v)=(\varsigma * \tau) * v$ for all $\varsigma, \tau, v \in[0,1]$;

(b) $\varsigma * 1=\varsigma$ for all $\varsigma \in I$;

(c) $\varsigma * \tau \leq v * \iota$ whenever $\varsigma \leq v$ and $\tau \leq \iota$ for all $\varsigma, \tau, v, \iota \in I$.

Some examples of $c t$-norms are as follows:

(1) $\varsigma *_{P} \tau=\varsigma \tau$;

(2) $\varsigma *_{M} \tau=\min \{\zeta, \tau\}$

(3) $\varsigma *_{L} \tau=\max \{\varsigma+\tau-1,0\}$ (the Lukasiewicz $t$-norm).

Definition 2.2 ([2]) Suppose that $*$ is a $c t$-norm, $V$ is a linear space and $\xi$ is a function from $V$ to $O^{+}$. The ordered tuple $(V, \xi, *)$ is called a Menger normed space (in short, $M N$ space) if the following conditions are satisfied:

(MN1) $\xi_{t}^{v}=\vartheta_{t}^{0}$ for all $t>0$ if and only if $v=0$;

(MN2) $\xi_{t}^{\alpha \nu}=\xi_{\frac{t}{|\alpha|}}^{v}$ for all $v \in V$ and $\alpha \in \mathbb{C}$ with $\alpha \neq 0$;

(MN3) $\xi_{t+s}^{u+v} \geq \xi_{t}^{u} * \xi_{s}^{v}$ for all $u, v \in V$ and $t, s \geq 0$.

A complete MN-space is called Menger Banach space, in short, MB-space. Let $(V,\|\cdot\|)$ be a normed space. Then

$$
\xi_{s}^{v}= \begin{cases}0, & \text { if } s \leq 0, \\ \exp \left(-\frac{\|v\|}{s}\right), & \text { if } s>0,\end{cases}
$$

defines a Menger norm and the ordered tuple $\left(V, \xi, *_{M}\right)$ is an MN-space. Also,

$$
\xi_{s}^{v}= \begin{cases}0, & \text { if } s \leq 0, \\ \frac{s}{s+\|v\|}, & \text { if } s>0,\end{cases}
$$

defines a Menger norm and the ordered tuple $\left(V, \xi, *_{M}\right)$ is an MN-space.

Definition $2.3([5,6])$ A Menger normed algebra (in short, MN-algebra) $(V, \xi, *, \star)$ is an $\mathrm{MN}$-space $(V, \xi, *)$ with algebraic structure such that 
(FN-5) $\xi_{t s}^{u v} \geq \xi_{t}^{u} \star \xi_{s}^{v}$ for all $u, v \in V$ and all $t, s>0$. in which $\star$ is a $c t$-norm.

Every normed algebra $(V,\|\cdot\|)$ defines an $\mathrm{MN}$-algebra $\left(V, \xi, *_{M}, *_{P}\right)$, where

$$
\xi_{s}^{v}= \begin{cases}0, & \text { if } s \leq 0, \\ \exp \left(-\frac{\|v\|}{s}\right), & \text { if } s>0,\end{cases}
$$

if and only if

$$
\|u v\| \leq\|u\|\|v\|+s\|v\|+t\|u\| \quad(u, v \in V ; t, s>0) .
$$

This space is called the induced MN-algebra. A complete MN-algebra is called Menger Banach algebra, in short, MB-algebra. Let $(\Gamma, \Sigma, \xi)$ be a probability measure space. Assume that $\left(T, \mathfrak{B}_{T}\right)$ and $\left(S, \mathfrak{B}_{S}\right)$ are Borel measurable spaces, in which $T$ and $S$ are complete MNspaces. A mapping $F: \Gamma \times T \rightarrow S$ is said to be a random operator if $\{\gamma: F(\gamma, t) \in B\} \in \Sigma$ for all $t$ in $T$ and $B \in \mathfrak{B}_{S}$. Also, $F$ is a random operator if $F(\gamma, t)=s(\gamma)$ is an $S$-valued random variable for all $t$ in $T$. A random operator $F: \Gamma \times T \rightarrow S$ is called linear if $F\left(\gamma, \alpha t_{1}+\beta t_{2}\right)=\alpha F\left(\gamma, t_{1}\right)+\beta F\left(\gamma, t_{2}\right)$ almost everywhere for $t_{1}, t_{2} \in T$ and $\alpha, \beta$ scalars, and bounded if there is a nonnegative random variable $M(\gamma)$ such that

$$
\xi_{M(\gamma) \tau}^{F(\gamma, t)-F(\gamma, s)} \geq \xi_{\tau}^{t-s}
$$

almost everywhere for each $t, s \in T$ and $\tau>0$.

Let $T$ be an MB-algebra. A linear random operator $\pi: \Gamma \times T \rightarrow T$ that satisfies

$$
\pi(\gamma, t s)=\pi(\gamma, t) s+t \pi(\gamma, s)
$$

for all $t, s \in T$ and $\gamma \in \Gamma$, is called stochastic derivation.

We denote by $\Pi(\Gamma, T)$ the set of $\mathbb{C}$-linear bounded stochastic derivations on $\Gamma \times T$. For $\pi_{1}, \pi_{2} \in \Pi(\Gamma, T)$

$$
\begin{aligned}
& \pi_{1} o \pi_{2}(\gamma, t s)=\pi_{1} o \pi_{2}(\gamma, t) s+\pi_{2}(\gamma, t) \pi_{1}(\gamma, s)+\pi_{1}(\gamma, t) \pi_{2}(\gamma, s)+t \pi_{1} o \pi_{2}(\gamma, s), \\
& \pi_{2} o \pi_{1}(\gamma, t s)=\pi_{2} o \pi_{1}(\gamma, t) s+\pi_{1}(\gamma, t) \pi_{2}(\gamma, s)+\pi_{2}(\gamma, t) \pi_{1}(\gamma, t)+t \pi_{2} o \pi_{1}(\gamma, s),
\end{aligned}
$$

for all $t, s \in T$ and $\gamma \in \Gamma$. Assume that $\left[\pi_{1}, \pi_{2}\right]=\pi_{1} o \pi_{2}-\pi_{2} o \pi_{1}$. Then

$$
\left[\pi_{1}, \pi_{2}\right](\gamma, t s)=\left[\pi_{1}, \pi_{2}\right](\gamma, t) s+t\left[\pi_{1}, \pi_{2}\right](\gamma, s)
$$

for all $t, s \in T$ and $\gamma \in \Gamma$. The $\mathbb{C}$-linearity of $\left[\pi_{1}, \pi_{2}\right]$ implies that $\left[\pi_{1}, \pi_{2}\right] \in \Pi(\Gamma, T)$ for all $\pi_{1}, \pi_{2} \in \Pi(\Gamma, T)$. Then $\Pi(\Gamma, T)$ is a stochastic Lie algebra with stochastic Lie bracket $\left[\pi_{1}, \pi_{2}\right], \pi_{1}+\pi_{2}$ and $\beta \pi_{1}$ are $\mathbb{C}$-linear stochastic derivations in which $\beta \in \mathbb{C}$.

Definition 2.4 Consider an MB-algebra $T$ and linear random operators $\Theta, \Phi: \Gamma \times$ $T \rightarrow T$. Set $[\Theta, \Phi](\gamma, t)=\Theta(\gamma, \Phi(\gamma, t))-\Phi(\gamma, \Theta(\gamma, t))$ for every $t \in T$ and $\gamma \in \Gamma$. The 
linear operator $[\Theta, \Phi]: \Gamma \times T \rightarrow T$ is said a stochastic Lie bracket (derivation, derivation) when

$$
\begin{aligned}
& {[\Theta, \Phi](\gamma, t s)=[\Theta, \Phi](\gamma, t) s+t[\Theta, \Phi](\gamma, s),} \\
& \Phi(\gamma, t s)=\Phi(\gamma, t) s+t \Phi(\gamma, s),
\end{aligned}
$$

for all $t, s \in T$ and $\gamma \in \Gamma$.

Recently, some authors have published some papers on approximation of functional equations in various spaces by the direct technique and the fixed point technique, for example, fuzzy Menger normed algebras [5], fuzzy metric spaces [7], fuzzy normed spaces [8], non-Archimedian random Lie $C^{*}$-algebras [9], random multi-normed space [10], nonArchimedean random normed spaces [6]; see also [11-30].

Note that a $[0, \infty]$-valued metric is called a generalized metric.

Theorem 2.5 ([31-33]) Consider a complete generalized metric space $(T, \delta)$ and a strictly contractive function $\Lambda: T \rightarrow T$ with Lipschitz constant $\beta<1$. Then, for every given element $t \in T$, either

$$
\delta\left(\Lambda^{n} t, \Lambda^{n+1} t\right)=\infty
$$

for each $n \in \mathbb{N}$ or there is an $n_{0} \in \mathbb{N}$ such that

(1) $\delta\left(\Lambda^{n} t, \Lambda^{n+1} t\right)<\infty$, for all $n \geq n_{0}$;

(2) the sequence $\left\{\Lambda^{n} t\right\}$ converges to a fixed point $s^{*}$ of $\Lambda$;

(3) $s^{*}$ is the unique fixed point of $\Lambda$ in the set $V=\left\{s \in T \mid \delta\left(\Lambda^{n_{0}} t, s\right)<\infty\right\}$;

(4) $(1-\beta) \delta\left(s, s^{*}\right) \leq \delta(s, \Lambda s)$ for every $s \in V$.

\section{Stability of (additive, additive) $(\omega, v)$-random operator inequality: direct technique}

Hereinafter we suppose that $*=*_{M}$.

Lemma 3.1 Assume thatrandom operators $G, H: \Gamma \times T \rightarrow T$ satisfy $G(\gamma, 0)=H(\gamma, 0)=0$ and

$$
\begin{aligned}
& \xi_{\tau}^{G(\gamma, t+s)-G(\gamma, t)-G(\gamma, s)} * \xi_{\tau}^{H(\gamma, t+s)+H(\gamma, t-s)-2 H(\gamma, t)} \\
& \quad \geq \xi_{\tau}^{\omega\left(2 G\left(\gamma, \frac{t+s}{2}\right)-G(\gamma, t)-G(\gamma, s)\right)} * \xi_{\tau}^{\nu\left(2 H\left(\gamma, \frac{t+s}{2}\right)+2 H\left(\gamma, \frac{t-s}{2}\right)-2 H(\gamma, t)\right)}
\end{aligned}
$$

for all $t, s \in T, \gamma \in \Gamma$ and $\tau>0$ in which $|\nu|<1$ and $|\omega|<1$. Then the random operators G, $H: \Gamma \times T \rightarrow T$ are additive.

Proof Putting $s=t$ in (3.1), we get

$$
\xi_{\tau}^{G(\gamma, 2 t)-2 G(\gamma, t)} * \xi_{\tau}^{H(\gamma, 2 t)-2 H(\gamma, t)} \geq \vartheta_{\tau}^{0}
$$


for all $t \in T$ and $\gamma \in \Gamma$. Then $G(\gamma, 2 t)=2 G(\gamma, t)$ and $H(\gamma, 2 t)=2 H(\gamma, t)$ for all $t \in T$ and $\gamma \in \Gamma$. By (3.1) we have

$$
\begin{aligned}
& \xi_{\tau}^{G(\gamma, t+s)-G(\gamma, t)-G(\gamma, s)} * \xi_{\tau}^{H(\gamma, t+s)+H(\gamma, t-s)-2 H(\gamma, t)} \\
& \quad \geq \xi_{\tau}^{\omega(G(\gamma, t+s)-G(\gamma, t)-G(\gamma, s))} * \xi_{\tau}^{\nu(H(\gamma, t+s)+H(\gamma, t-s)-2 H(\gamma, t))}
\end{aligned}
$$

for all $t, s \in T, \gamma \in \Gamma$ and $\tau>0$. So $|\nu|<1$ and $|\omega|<1$ imply that $G(\gamma, t+s)-G(\gamma, t)-$ $G(\gamma, s)=0$ and $H(\gamma, t+s)+H(\gamma, t-s)-2 H(\gamma, t)=0$ for all $t \in T$ and $\gamma \in \Gamma$. Thus the random operators $G, H: \Gamma \times T \rightarrow T$ are additive.

Lemma 3.2 ([34, Theorem 2.1]) Assume that a random operator $F: \Gamma \times T \rightarrow T$ is additive and

$$
F(\gamma, d t)=d F(\gamma, t)
$$

for all $d \in \mathbb{D}^{1}:=\{c \in \mathbb{C}:|c|=1\}$ and each $t \in T$ and $\gamma \in \Gamma$. Then the random operator $F: \Gamma \times T \rightarrow T$ is $\mathbb{C}$-linear.

Theorem 3.3 Let $(T, \xi, *, *)$ be an MB-algebra. Let $\varphi: T^{2} \rightarrow O^{+}$be a distribution function such that there exists $a \beta \in(0,1)$ with

$$
\varphi_{\frac{\beta}{2} \tau}^{\frac{t}{2}, \frac{s}{2}} \geq \varphi_{\frac{\beta}{4} \tau}^{\frac{t}{2}, \frac{s}{2}} \geq \varphi_{\tau}^{t, s}
$$

for all $t, s \in T$ and $\tau>0$. Suppose that random operators $G, H: \Gamma \times T \rightarrow T$ satisfy $G(\gamma, 0)=$ $H(\gamma, 0)=0$ and

$$
\begin{aligned}
& \xi_{\tau}^{G(\gamma, d(t+s))-d G(\gamma, t)-d G(\gamma, s)} * \xi_{\tau}^{H(\gamma, d(t+s))+H(\gamma, d(t-s))-2 d H(\gamma, t)} \\
& \geq \xi_{\tau}^{\omega\left(2 G\left(\gamma, d \frac{t+s}{2}\right)-d G(\gamma, t)-d G(\gamma, s)\right)} \\
& \quad * \xi_{\tau}^{\nu\left(2 H\left(\gamma, d \frac{t+s}{2}\right)+2 H\left(\gamma, d \frac{t-s}{2}\right)-2 d H(\gamma, t)\right)} * \varphi_{\tau}^{t, s}
\end{aligned}
$$

for all $d \in \mathbb{D}^{1}, t, s \in T, \gamma \in \Gamma$ and $\tau>0$. Assume that the random operators $G, H: \Gamma \times T \rightarrow$ T satisfy

$$
\xi_{\tau}^{[G, H](\gamma, t s)-[G, H](\gamma, t) s-t[G, H](\gamma, s)} * \xi_{\tau}^{H(\gamma, t s)-H(\gamma, t) s-t H(\gamma, s)} \geq \varphi_{\tau}^{t, s}
$$

for all $t, s \in T, \gamma \in \Gamma$ and $\tau>0$. Then there are a unique $\mathbb{C}$-linear random operator $\Theta: \Gamma \times$ $T \rightarrow T$ and a unique stochastic derivation $\pi: \Gamma \times T \rightarrow T$ such that $[\Theta, \pi]: \Gamma \times T \rightarrow T$ is a stochastic derivation and

$$
\xi_{\tau}^{G(\gamma, t)-\Theta(\gamma, t)} * \xi_{\tau}^{H(\gamma, t)-\pi(\gamma, t)} \geq \varphi_{\frac{2(1-\beta)}{\beta} \tau}^{t, t}
$$

for all $t \in T, \gamma \in \Gamma$ and $\tau>0$. 
Proof In (3.3), putting $d=1$ and $s=t$, one obtains

$$
\xi_{\tau}^{G(\gamma, 2 t)-2 G(\gamma, t)} * \xi_{\tau}^{H(\gamma, 2 t)-2 H(\gamma, t)} \geq \varphi_{\tau}^{t, t}
$$

and so

$$
\begin{aligned}
\xi_{\tau}^{G(\gamma, t)-2 G\left(\gamma, \frac{t}{2}\right)} * \xi_{\tau}^{H(\gamma, t)-2 H\left(\gamma, \frac{t}{2}\right)} & \geq \varphi_{\tau}^{\frac{t}{2}, \frac{t}{2}} \\
& \geq \varphi_{\frac{2}{\beta} \tau}^{t, t}
\end{aligned}
$$

for all $t \in T, \gamma \in \Gamma$ and $\tau>0$. Replacing $t$ by $\frac{t}{2^{n}}$ in (3.7), we get

$$
\begin{aligned}
\xi_{\tau}^{2^{n} G\left(\gamma, \frac{t}{2^{n}}\right)-2^{n+1} G\left(\gamma, \frac{t}{2^{n+1}}\right)} * \xi_{\tau}^{2^{n} H\left(\gamma, \frac{t}{2^{n}}\right)-2^{n+1} H\left(\gamma, \frac{t}{2^{n+1}}\right)} & \geq \varphi_{\frac{2}{\beta} \tau}^{\frac{t}{2^{n+1}}, \frac{t}{2^{n+1}}} \\
& \geq \varphi_{\frac{2}{\beta^{n+1}} \tau}^{t, t}
\end{aligned}
$$

for all $t \in T, \gamma \in \Gamma, \tau>0$ and $n \in \mathbb{N}$. Since

$$
2^{n} G\left(\gamma, \frac{t}{2^{n}}\right)-G(\gamma, t)=\sum_{k=1}^{n} 2^{k} G\left(\gamma, \frac{t}{2^{k}}\right)-2^{k-1} G\left(\gamma, \frac{t}{2^{k-1}}\right),
$$

we have

$$
\begin{aligned}
& \xi_{\sum_{k=1}^{n} \frac{1}{2} \beta^{k} \tau}^{2^{n} G\left(\gamma, \frac{t}{2^{n}}\right)-G(\gamma, t)} * \xi_{\sum_{k=1}^{n} \frac{1}{2} \beta^{k} \tau}^{2^{n} H\left(\gamma, \frac{t}{2^{n}}\right)-H(\gamma, t)} \\
& \quad \geq \prod_{k=1}^{n}\left[\xi_{\frac{1}{2} \beta^{k} \tau}^{2^{k} G\left(\gamma, \frac{t}{2^{k}}\right)-2^{k-1} G\left(\gamma, \frac{t}{2^{k-1}}\right)} * \xi_{\frac{1}{2} \beta^{k} \tau}^{2^{k} H\left(\gamma, \frac{t}{2^{k}}\right)-2^{k-1} H\left(\gamma, \frac{t}{2^{k-1}}\right)}\right] \\
& \quad \geq \varphi_{\tau}^{t, t}
\end{aligned}
$$

and so

$$
\xi_{\tau}^{2^{n} G\left(\gamma, \frac{t}{2^{n}}\right)-G(\gamma, t)} * \xi_{\tau}^{2^{n} H\left(\gamma, \frac{t}{2^{n}}\right)-H(\gamma, t)} \geq \varphi_{\frac{t, t}{\sum_{k=1}^{n} \frac{1}{2} \beta^{k}}}
$$

for all $t \in T, \gamma \in \Gamma, \tau>0$ and $n \in \mathbb{N}$.

Replacing $t$ by $\frac{t}{2^{m}}$ in (3.10), we get

$$
\begin{aligned}
& \xi_{\tau}^{2^{n+m} G\left(\gamma, \frac{t}{2^{n+m}}\right)-2^{m} G\left(\gamma, \frac{t}{2^{m}}\right)} * \xi_{\tau}^{2^{n+m} H\left(\gamma, \frac{t}{2^{n+m}}\right)-2^{m} H\left(\gamma, \frac{t}{2^{n+m}}\right)} \geq \frac{\varphi^{\frac{t}{2^{m}}, \frac{t}{2^{m}}}}{\sum_{k=1}^{2^{m}} \frac{1}{2} \beta^{k}} \\
& \geq \varphi \frac{t, t}{\sum_{k=m+1}^{n+m} \frac{1}{2} \beta^{k}},
\end{aligned}
$$

for all $t \in T, \gamma \in \Gamma, \tau>0$ and $n, m \in \mathbb{N}$.

Let $m, n \rightarrow \infty$ in (3.11), since $\beta \in(0,1)$, we conclude that $\varphi_{\frac{\tau}{\sum_{k=m+1}^{n+m} \frac{1}{2} \beta^{k}}}$ tends to 1 for all $\tau>0$. Thus this shows that $\left\{2^{n} G\left(\gamma, \frac{t}{2^{n}}\right)\right\}$ and $\left\{2^{n} H\left(\gamma, \frac{t}{2^{n}}\right)\right\}$ are Cauchy sequences for each 
$t \in T, \gamma \in \Gamma$. Since $T$ is complete, the mentioned sequences converge. Now we define the random operators $\Theta, \pi: \Gamma \times T \rightarrow T$ by

$$
\Theta(\gamma, t):=\lim _{n \rightarrow+\infty} 2^{n} G\left(\gamma, \frac{t}{2^{n}}\right), \quad \pi(\gamma, t):=\lim _{n \rightarrow+\infty} 2^{n} H\left(\gamma, \frac{t}{2^{n}}\right)
$$

for each $t \in T, \gamma \in \Gamma$. Putting $m=0$ and $n \rightarrow+\infty$ in (3.11), we obtain (3.5).

Using (3.3), (3.12) and letting $n$ tend to $+\infty$, we have

$$
\begin{aligned}
& \xi_{\tau}^{\Theta(\gamma, d(t+s))-d \Theta(\gamma, t)-d \Theta(\gamma, s)} * \xi_{\tau}^{\pi(\gamma, d(t+s))+\pi(\gamma, d(t-s))-2 d \pi(\gamma, s)} \\
& \quad=\xi_{\frac{\tau}{2^{n}}}^{G\left(\gamma, d\left(\frac{t+s}{2^{n}}\right)\right)-d G\left(\gamma, \frac{t}{2^{n}}\right)-d G\left(\gamma, \frac{t}{2^{n}}\right)} * \xi_{\frac{\tau}{2^{n}}}^{H\left(\gamma, d\left(\frac{t+s}{2^{n}}\right)\right)+H\left(\gamma, d\left(\frac{t-s}{2^{n}}\right)\right)-2 d H\left(\gamma, \frac{s}{2^{n}}\right)} \\
& \quad \geq \xi_{\frac{\tau}{2^{n}}}^{\omega\left(2 G\left(\gamma, d \frac{t+s}{2^{n+1}}\right)-d G\left(\gamma, \frac{t}{2^{n}}\right)-d G\left(\gamma, \frac{s}{2^{n}}\right)\right)} * \xi_{\frac{\tau}{2^{n}}}^{\nu\left(2 H\left(\gamma, d \frac{t+s}{2^{n+1}}\right)+2 H\left(\gamma, d \frac{t-s}{2^{n+1}}\right)-2 d H\left(\gamma, \frac{t}{2^{n}}\right)\right)} * \varphi_{\frac{\tau}{2^{n}}}^{\frac{t}{2^{n}}, \frac{s}{2^{n}}} \\
& \quad \geq \xi_{\tau}^{\omega\left(2 \Theta\left(\gamma, d \frac{t+s}{2}\right)-d \Theta(\gamma, t)-d \Theta(\gamma, s)\right)} * \xi_{\tau}^{\nu\left(2 \pi\left(\gamma, d \frac{t+s}{2}\right)+2 \pi\left(\gamma, d \frac{t-s}{2}\right)-2 d \pi(\gamma, s)\right)}
\end{aligned}
$$

for all $d \in \mathbb{D}^{1}, t, s \in T, \gamma \in \Gamma$ and $\tau>0$. Then

$$
\begin{aligned}
& \xi_{\tau}^{\Theta(\gamma, d(t+s))-d \Theta(\gamma, t)-d \Theta(\gamma, s)} * \xi_{\tau}^{\pi(\gamma, d(t+s))+\pi(\gamma, d(t-s))-2 d \pi(\gamma, s)} \\
& \quad \geq \xi_{\tau}^{\omega\left(2 \Theta\left(\gamma, d \frac{t+s}{2}\right)-d \Theta(\gamma, t)-d \Theta(\gamma, s)\right)} * \xi_{\tau}^{\nu\left(2 \pi\left(\gamma, d \frac{t+s}{2}\right)+2 \pi\left(\gamma, d \frac{t-s}{2}\right)-2 d \pi(\gamma, s)\right)}
\end{aligned}
$$

for all $d \in \mathbb{D}^{1}$ and $t, s \in T, \gamma \in \Gamma, \tau>0$. Putting $d=1$ in (3.13) and using Lemma 3.1, we see that the random operators $\Theta, \pi: \Gamma \times T \rightarrow T$ are additive.

The additivity of $\Theta$ and $\pi$ and (3.13) imply that

$$
\begin{aligned}
& \xi_{\tau}^{\Theta(\gamma, d(t+s))-d \Theta(\gamma, t)-d \Theta(\gamma, s)} * \xi_{\tau}^{\pi(\gamma, d(t+s))+\pi(\gamma, d(t-s))-2 d \pi(\gamma, s)} \\
& \quad \geq \xi_{\tau}^{\omega(\Theta(\gamma, d(t+s))-d \Theta(\gamma, t)-d \Theta(\gamma, s))} * \xi_{\tau}^{\nu(\pi(\gamma, d(t+s))+\pi(\gamma, d(t-s))-2 d \pi(\gamma, s))}
\end{aligned}
$$

for all $d \in \mathbb{D}^{1}$ and $t, s \in T, \gamma \in \Gamma, \tau>0$, which implies that

$$
\begin{aligned}
& \Theta(\gamma, d(t+s))-d \Theta(\gamma, t)-d \Theta(\gamma, s)=0, \\
& \pi(\gamma, d(t+s))+\pi(\gamma, d(t-s))-2 d \pi(\gamma, s)=0 .
\end{aligned}
$$

Then $\Theta(\gamma, d t)=d \Theta(\gamma, t)$ and $\pi(\gamma, d t)=d \pi(\gamma, t)$ for all $d \in \mathbb{D}^{1}$ and $t \in T, \gamma \in \Gamma$. Now, Lemma 3.2 implies that the additive mappings $\Theta$ and $\pi$ are $\mathbb{C}$-linear.

The additivity of $\Theta$ and $\pi$ and (3.4) imply that

$$
\begin{aligned}
& \xi_{\tau}^{[\Theta, \phi](\gamma, t s)-[\Theta, \phi](\gamma, t) s-t[\Theta, \phi](\gamma, s)} * \xi_{\tau}^{\pi(\gamma, t s)-\pi(\gamma, t) s-t \pi(\gamma, s)} \\
& \geq \xi_{\frac{\tau}{4^{n}}}^{[G, H]\left(\gamma, \frac{t s}{4^{n}}\right)-[G, H]\left(\gamma, \frac{t}{2^{n}}\right) \frac{s}{2^{n}}-\frac{t}{2^{n}}[G, H]\left(\gamma, \frac{s}{2^{n}}\right)} * \xi_{\frac{\tau}{4^{n}}}^{H\left(\gamma, \frac{t s}{4^{n}}\right)-H\left(\gamma, \frac{t}{2^{n}}\right) \frac{s}{2^{n}}-\frac{t}{2^{n}} H\left(\gamma, \frac{s}{2^{n}}\right)} \\
& \geq \varphi_{\frac{\tau}{4^{n}}}^{\frac{t}{2^{n}} \frac{s}{2^{n}}} \geq \varphi_{\frac{\tau}{\beta^{n}}}^{t, t}
\end{aligned}
$$

which tends to 1 as $n \rightarrow+\infty$. Then

$$
[\Theta, \phi](\gamma, t s)-[\Theta, \phi](\gamma, t) s-t[\Theta, \phi](\gamma, s)=0,
$$




$$
\pi(\gamma, t s)-\pi(\gamma, t) s-t \pi(\gamma, s)=0
$$

for all $t, s \in T, \gamma \in \Gamma$. Thus $[\Theta, \phi]$ and $\pi$ are stochastic derivations.

Corollary 3.4 Let $(T, \xi, *, *)$ be an MB-algebra. Assume that $q>0$ and $p>1$. Suppose that random operators $G, H: \Gamma \times T \rightarrow T$ satisfy $G(\gamma, 0)=H(\gamma, 0)=0$ and

$$
\begin{aligned}
& \xi_{\tau}^{G(\gamma, d(t+s))-d G(\gamma, t)-d G(\gamma, s)} * \xi_{\tau}^{H(\gamma, d(t+s))+H(\gamma, d(t-s))-2 d H(\gamma, t)} \\
& \quad \geq \xi_{\tau}^{\omega\left(2 G\left(\gamma, d \frac{t+s}{2}\right)-d G(\gamma, t)-d G(\gamma, s)\right)} \\
& \quad * \xi_{\tau}^{\nu\left(2 H\left(\gamma, d \frac{t+s}{2}\right)+2 H\left(\gamma, d \frac{t-s}{2}\right)-2 d H(\gamma, t)\right)} * \frac{\tau}{\tau+q\left(\|t\|^{p}+\|s\|^{p}\right)}
\end{aligned}
$$

for all $d \in \mathbb{D}^{1}, t, s \in T, \gamma \in \Gamma$ and $\tau>0$. Let

$$
\xi_{\tau}^{[G, H](\gamma, t s)-[G, H](\gamma, t) s-t[G, H](\gamma, s)} * \xi_{\tau}^{H(\gamma, t s)-H(\gamma, t) s-t H(\gamma, s)} \geq \frac{\tau}{\tau+q\left(\|t\|^{p}+\|s\|^{p}\right)}
$$

for all $t, s \in T, \gamma \in \Gamma$ and $\tau>0$. Then there are a unique $\mathbb{C}$-linear random operator $\Theta: \Gamma \times$ $T \rightarrow T$ and a unique stochastic derivation $\pi: \Gamma \times T \rightarrow T$ such that $[\Theta, \pi]: \Gamma \times T \rightarrow T$ is a stochastic derivation and

$$
\xi_{\tau}^{G(\gamma, t)-\Theta(\gamma, t)} * \xi_{\tau}^{H(\gamma, t)-\pi(\gamma, t)} \geq \frac{\tau}{\tau+q\left(\frac{2}{2^{p}-2}\|t\|^{p}\right)}
$$

for all $t \in T, \gamma \in \Gamma$ and $\tau>0$.

Proof In Theorem 3.3, putting

$$
\varphi_{\tau}^{t, s}=\frac{\tau}{\tau+q\left(\|t\|^{p}+\|s\|^{p}\right)}
$$

and letting $\beta=2^{1-p}$, we get the desired result.

Theorem 3.5 Let $(T, \xi, *, *)$ be an MB-algebra. Let $\varphi: T^{2} \rightarrow O^{+}$be a distribution function such that there exists a $\beta \in(0,1)$ with

$$
\varphi_{4 \beta \tau}^{t, s} \geq \varphi_{\tau}^{\frac{t}{2}, \frac{s}{2}}
$$

for all $t, s \in T$ and $\tau>0$. Suppose that the random operators $G, H: \Gamma \times T \rightarrow T$ satisfy $G(\gamma, 0)=H(\gamma, 0)=0$, (3.3) and (3.4). Then there are a unique $\mathbb{C}$-linear random operator $\Theta: \Gamma \times T \rightarrow T$ and a unique stochastic derivation $\pi: \Gamma \times T \rightarrow T$ such that $[\Theta, \pi]$ : $\Gamma \times T \rightarrow T$ is a stochastic derivation and

$$
\xi_{\tau}^{G(\gamma, t)-\Theta(\gamma, t)} * \xi_{\tau}^{H(\gamma, t)-\pi(\gamma, t)} \geq \varphi_{2(1-\beta) \tau}^{t, t}
$$

for all $t \in T, \gamma \in \Gamma$ and $\tau>0$. 
Proof Using (3.6), we get

$$
\xi_{\tau}^{G(\gamma, t)-\frac{1}{2} G(\gamma, 2 t)} * \xi_{\tau}^{H(\gamma, t)-\frac{1}{2} H(\gamma, 2 t)} \geq \varphi_{2 \tau}^{2 t, 2 t} \geq \varphi_{\frac{\tau}{2 \beta}}^{t, t}
$$

for all $t \in T, \gamma \in \Gamma$ and $\tau>0$.

Replacing $t$ by $2^{n} t$ in (3.21), we get

$$
\begin{aligned}
\xi_{\tau}^{\frac{1}{2^{n}} G\left(\gamma, 2^{n} t\right)-\frac{1}{2^{n+1}} G\left(\gamma, 2^{n+1} t\right)} * \xi_{\tau}^{\frac{1}{2^{n}} H\left(\gamma, 2^{n} t\right)-\frac{1}{2^{n+1}} H\left(\gamma, 2^{n+1} t\right)} & \geq \varphi_{2^{2^{n+1}} \tau}^{2^{n+1}, 2^{n+1} t} \\
& \geq \varphi_{\frac{2^{n+1}}{(4 \beta)^{n}} \tau}
\end{aligned}
$$

for all $t \in T, \gamma \in \Gamma, \tau>0$ and $n \in \mathbb{N}$. Since

$$
\frac{1}{2^{n}} G\left(\gamma, 2^{n} t\right)-G(\gamma, t)=\sum_{k=0}^{n-1} \frac{1}{2^{k+1}} G\left(\gamma, 2^{k+1} t\right)-\frac{1}{2^{k}} G\left(\gamma, 2^{k} t\right)
$$

we have

$$
\begin{aligned}
& \xi^{\frac{1}{2^{n}} G\left(\gamma, 2^{n} t\right)-G(\gamma, t)} * \xi_{k=0}^{\frac{1}{2^{n}} H\left(\gamma, 2^{n} t\right)-H(\gamma, t)} \\
& \geq \prod_{k=0}^{n-1}\left[\begin{array}{l}
\xi^{\frac{1}{2^{k+1}}} G\left(\gamma, 2^{k+1} t\right)-\frac{1}{2^{k}} G\left(\gamma, 2^{k} t\right) \\
\frac{(4 \beta)^{k}}{2^{k+1}} \tau
\end{array} \xi_{\frac{(4 \beta)^{k}}{2^{k+1}} \tau}^{\frac{1}{2^{k+1}} H\left(\gamma, 2^{k+1} t\right)-\frac{1}{2^{k}} H\left(\gamma, 2^{k} t\right)}\right] \\
& \geq \varphi_{\tau}^{t, t}
\end{aligned}
$$

and so

$$
\xi_{\tau}^{\frac{1}{2^{n}} G\left(\gamma, 2^{n} t\right)-G(\gamma, t)} * \xi_{\tau}^{\frac{1}{2^{n}} H\left(\gamma, 2^{n} t\right)-H(\gamma, t)} \geq \varphi^{t, t} \frac{\tau}{\sum_{k=0}^{n-1} \frac{(4 \beta)^{k}}{2^{k+1}}}
$$

for all $t \in T, \gamma \in \Gamma, \tau>0$ and $n \in \mathbb{N}$.

Replacing $t$ by $2^{m} t$ in (3.24), we get

$$
\begin{aligned}
& \xi_{\tau}^{\frac{1}{2^{n+m}} G\left(\gamma, 2^{n+m} t\right)-\frac{1}{2^{m}} G\left(\gamma, 2^{m} t\right)} * \xi_{\tau}^{\frac{1}{2^{n+m}} H\left(\gamma, 2^{n+m} t\right)-\frac{1}{2^{m}} H\left(\gamma, 2^{m} t\right)} \geq \varphi^{2^{m} t, 2^{m} t} \\
& \geq \varphi_{\frac{1}{2^{m} \tau}}^{\sum_{k=0}^{n-1} \frac{(4 \beta)^{k}}{2^{k+1}}} \\
& \sum_{k=m}^{n+t} \frac{\tau}{2^{k+1}}
\end{aligned}
$$

for all $t \in T, \gamma \in \Gamma, \tau>0$ and $n, m \in \mathbb{N}$.

Letting $m, n \rightarrow+\infty$ in (3.25), since $\beta \in(0,1)$, we conclude that $\varphi_{\frac{\tau}{\sum_{k=m}^{n+m} \frac{(4 \beta)^{k}}{2^{k+1}}}}$ tends to 1 for all $\tau>0$. This shows that $\left\{\frac{1}{2^{n}} G\left(\gamma, 2^{n} t\right)\right\}$ and $\left\{\frac{1}{2^{n}} H\left(\gamma, 2^{n} t\right)\right\}$ are Cauchy sequences for each $t \in T, \gamma \in \Gamma$. Since $T$ is complete, the mentioned sequences converge. Now we define the random operators $\Theta, \pi: \Gamma \times T \rightarrow T$ by

$$
\Theta(\gamma, t):=\lim _{n \rightarrow+\infty} \frac{1}{2^{n}} G\left(\gamma, 2^{n} t\right), \quad \pi(\gamma, t):=\lim _{n \rightarrow+\infty} \frac{1}{2^{n}} G\left(\gamma, 2^{n} t\right)
$$


for each $t \in T, \gamma \in \Gamma$. Putting $m=0$ and $n \rightarrow \infty$ in (3.25), we get (3.5). By the same method in the proof of Theorem 3.3, the random operators $\Theta, \pi: \Gamma \times T \rightarrow T$ are $\mathbb{C}$-linear.

The additivity of $\Theta$ and $\pi$ and (3.4) imply that

$$
\begin{aligned}
& \xi_{\tau}^{[\Theta, \phi](\gamma, t s)-[\Theta, \phi](\gamma, t) s-t[\Theta, \phi](\gamma, s)} * \xi_{\tau}^{\pi(\gamma, t s)-\pi(\gamma, t) s-t \pi(\gamma, s)} \\
& \quad \geq \xi_{4^{n} \tau}^{[G, H]\left(\gamma, 4^{n} t s\right)-[G, H]\left(\gamma, 2^{n} t\right) 2^{n} s-2^{n} t[G, H]\left(\gamma, 2^{n} s\right)} * \xi_{4^{n} \tau}^{H\left(\gamma, 4^{n} t s\right)-H\left(\gamma, 2^{n} t\right) 2^{n} s-2^{n} t H\left(\gamma, 2^{n} s\right)} \\
& \quad \geq \varphi_{4^{n} \tau}^{2^{n} t, 2^{n} s} \\
& \quad \geq \varphi_{\frac{\tau}{\beta^{n}}}^{t, t}
\end{aligned}
$$

which tends to 1 as $n \rightarrow+\infty$. Then

$$
\begin{aligned}
& {[\Theta, \phi](\gamma, t s)-[\Theta, \phi](\gamma, t) s-t[\Theta, \phi](\gamma, s)=0,} \\
& \pi(\gamma, t s)-\pi(\gamma, t) s-t \pi(\gamma, s)=0
\end{aligned}
$$

for all $t, s \in T, \gamma \in \Gamma$. Thus $[\Theta, \phi]$ and $\pi$ are stochastic derivations.

Corollary 3.6 Let $(T, \xi, *, *)$ be an $M B$-algebra. Assume that $q>0$ and $p<1$. Suppose that random operators $G, H: \Gamma \times T \rightarrow T$ satisfy $G(\gamma, 0)=H(\gamma, 0)=0,(3.16)$ and (3.17). Then there are a unique $\mathbb{C}$-linear random operator $\Theta: \Gamma \times T \rightarrow T$ and a unique stochastic derivation $\pi: \Gamma \times T \rightarrow T$ such that $[\Theta, \pi]: \Gamma \times T \rightarrow T$ is a stochastic derivation and

$$
\xi_{\tau}^{G(\gamma, t)-\Theta(\gamma, t)} * \xi_{\tau}^{H(\gamma, t)-\pi(\gamma, t)} \geq \frac{\tau}{\tau+q\left(\frac{2}{2-2^{p}}\|t\|^{p}\right)}
$$

for all $t \in T, \gamma \in \Gamma$ and $\tau>0$.

Proof In Theorem 3.5, putting

$$
\varphi_{\tau}^{t, s}=\frac{\tau}{\tau+q\left(\|t\|^{p}+\|s\|^{p}\right)}
$$

and letting $\beta=2^{p-1}$, we get the desired result.

\section{Stability of (additive, additive) $(\omega, v)$-random operator inequality (1.1) via fixed point technique}

Theorem 4.1 Let $(T, \xi, *, *)$ be an $M B$-algebra. Let $\varphi: T^{2} \rightarrow O^{+}$be a distribution function such that there exists a $\beta \in(0,1)$ with

$$
\varphi_{\frac{\beta}{2} \tau}^{\frac{t}{2}, \frac{s}{2}} \geq \varphi_{\frac{\beta}{4} \tau}^{\frac{t}{2}, \frac{s}{2}} \geq \varphi_{\tau}^{t, s}
$$

for all $t, s \in T$ and $\tau>0$. Suppose that random operators $G, H: \Gamma \times T \rightarrow T$ satisfy $G(\gamma, 0)=$ $H(\gamma, 0)=0$, (3.3) and (3.4). Then there are a unique $\mathbb{C}$-linear random operator $\Theta: \Gamma \times$ $T \rightarrow T$ and a unique stochastic derivation $\pi: \Gamma \times T \rightarrow T$ such that $[\Theta, \pi]: \Gamma \times T \rightarrow T$ 
is a stochastic derivation and

$$
\xi_{\tau}^{G(\gamma, t)-\Theta(\gamma, t)} * \xi_{\tau}^{H(\gamma, t)-\pi(\gamma, t)} \geq \varphi_{\frac{2(1-\beta)}{\beta} \tau}^{t, t}
$$

for all $t \in T, \gamma \in \Gamma$ and $\tau>0$.

Proof By Theorem 3.3, there exist a unique $\mathbb{C}$-linear random operator $\Theta: \Gamma \times T \rightarrow T$ and a unique stochastic derivation $\pi: \Gamma \times T \rightarrow T$ such that $[\Theta, \pi]: \Gamma \times T \rightarrow T$ is a stochastic a derivation.

In (3.3), putting $d=1$ and $s=t$, we get

$$
\xi_{\tau}^{G(\gamma, 2 t)-2 G(\gamma, t)} * \xi_{\tau}^{H(\gamma, 2 t)-2 H(\gamma, t)} \geq \varphi_{\tau}^{t, t}
$$

and so

$$
\begin{aligned}
\xi_{\tau}^{G(\gamma, t)-2 G\left(\gamma, \frac{t}{2}\right)} * \xi_{\tau}^{H(\gamma, t)-2 H\left(\gamma, \frac{t}{2}\right)} & \geq \varphi_{\tau}^{\frac{t}{2}, \frac{t}{2}} \\
& \geq \varphi_{\frac{2}{\beta} \tau}^{t, t}
\end{aligned}
$$

for all $t \in T, \gamma \in \Gamma$ and $\tau>0$.

On the set

$$
S:=\{(G, H) \mid G, H: \Gamma \times T \rightarrow T, G(\gamma, 0)=H(\gamma, 0)=0\},
$$

we define the following generalized metric on $S$ :

$$
\begin{aligned}
& \delta\left((G, H),\left(G_{1}, H_{1}\right)\right) \\
& \quad=\inf \left\{\mu \in \mathbb{R}_{+}: \xi_{\tau}^{G(\gamma, t)-G_{1}(\gamma, t)} * \xi_{\tau}^{H(\gamma, t)-H_{1}(\gamma, t)} \geq \varphi_{\frac{\tau}{\mu}}^{t, t}, \forall t \in T, \gamma \in \Gamma, \tau>0\right\} .
\end{aligned}
$$

In [35], Miheț and Radu proved that $(S, \delta)$ is complete (see also [36]).

Now, we consider the linear mapping $\Lambda: S \rightarrow S$ such that

$$
\Lambda(G, H)(\gamma, t):=\left(2 G\left(\gamma, \frac{t}{2}\right), 2 H\left(\gamma, \frac{t}{2}\right)\right)
$$

for all $t \in T, \gamma \in \Gamma$.

Let $(G, H),\left(G_{1}, H_{1}\right) \in S$ be given such that $\delta\left((G, H),\left(G_{1}, H_{1}\right)\right)=\varepsilon$. Then

$$
\xi_{\tau}^{G(\gamma, t)-G_{1}(\gamma, t)} * \xi_{\tau}^{H(\gamma, t)-H_{1}(\gamma, t)} \geq \varphi_{\frac{\tau}{\varepsilon}}^{t, t}
$$

for all $t \in T, \gamma \in \Gamma$ and $\tau>0$. So

$$
\xi_{\tau}^{2 G\left(\gamma, \frac{t}{2}\right)-2 G_{1}\left(\gamma, \frac{t}{2}\right)} * \xi_{\tau}^{2 H\left(\gamma, \frac{t}{2}\right)-H_{1}\left(\gamma, \frac{t}{2}\right)} \geq \varphi_{\frac{\tau}{\varepsilon}}^{\frac{t}{2}, \frac{t}{2}} \geq \varphi_{\frac{\tau}{\beta \varepsilon}}^{t, t}
$$

for all $t \in T, \gamma \in \Gamma, \tau>0$ and $\delta\left(\Lambda(G, H), \Lambda\left(G_{1}, H_{1}\right)\right) \leq \beta \varepsilon$. This means that

$$
\delta\left(\Lambda(G, H), \Lambda\left(G_{1}, H_{1}\right)\right) \leq \beta \delta\left((G, H),\left(G_{1}, H_{1}\right)\right)
$$

for all $(G, H),\left(G_{1}, H_{1}\right) \in S$. 
It follows from (3.3) that

$$
\xi_{\tau}^{G(\gamma, t)-2 G_{1}\left(\gamma, \frac{t}{2}\right)} * \xi_{\tau}^{H(\gamma, t)-H_{1}\left(\gamma, \frac{t}{2}\right)} \geq \varphi_{\tau}^{\frac{t}{2}, \frac{t}{2}} \geq \varphi_{\frac{2 \tau}{\beta}}^{t, t}
$$

for all $t \in T, \gamma \in \Gamma$ and $\tau>0$. So $\delta((G, H), \Lambda(G, H)) \leq \frac{\beta}{2}$. By Theorem 2.5, there exist random operators $\Theta, \pi: \Gamma \times T \rightarrow T$ satisfying the following:

(1) There is a fixed point $(\Theta, \pi)$ for the function $\Lambda$ such that

$$
\Theta(\gamma, t):=2 \Theta\left(\gamma, \frac{t}{2}\right), \quad \pi(\gamma, t):=2 \pi\left(\gamma, \frac{t}{2}\right)
$$

for all $t \in T, \gamma \in \Gamma$. The random operator $(\Theta, \pi)$ is a unique fixed point of $\Lambda$ in the set

$$
M=\left\{(G, H) \in S: \delta\left((G, H),\left(G_{1}, H_{1}\right)\right)<\infty\right\}
$$

(2) $\delta\left(\Lambda^{n}(G, H),(\Theta, \pi)\right) \rightarrow 0$ as $n \rightarrow+\infty$. which implies

$$
\Theta(\gamma, t):=\lim _{n \rightarrow+\infty} 2^{n} G\left(\gamma, \frac{t}{2^{n}}\right), \quad \pi(\gamma, t):=\lim _{n \rightarrow+\infty} 2^{n} H\left(\gamma, \frac{t}{2^{n}}\right) .
$$

(3) $\delta((G, H),(\Theta, \pi)) \leq \frac{1}{1-\beta} \delta((G, H), \Lambda(G, H))$, which implies

$$
\xi_{\tau}^{G(\gamma, t)-\Theta(\gamma, t)} * \xi_{\tau}^{H(\gamma, t)-\pi(\gamma, t)} \geq \varphi_{\frac{2(1-\beta)}{\beta} \tau}^{t, t}
$$

for all $t \in T, \gamma \in \Gamma$ and $\tau>0$.

Corollary 4.2 Let $(T, \xi, *, *)$ be an MB-algebra. Assume that $q>0$ and $p>1$. Suppose that random operators $G, H: \Gamma \times T \rightarrow T$ satisfy $G(\gamma, 0)=H(\gamma, 0)=0$, (3.16) and (3.17). Then there are a unique $\mathbb{C}$-linear random operator $\Theta: \Gamma \times T \rightarrow T$ and a unique stochastic derivation $\pi: \Gamma \times T \rightarrow T$ such that $[\Theta, \pi]: \Gamma \times T \rightarrow T$ is a stochastic derivation and

$$
\xi_{\tau}^{G(\gamma, t)-\Theta(\gamma, t)} * \xi_{\tau}^{H(\gamma, t)-\pi(\gamma, t)} \geq \exp \left(-\frac{q\left(\frac{2}{2^{p}-2}\|t\|^{p}\right)}{\tau}\right)
$$

for all $t \in T, \gamma \in \Gamma$ and $\tau>0$.

Proof In Theorem 4.1, putting

$$
\varphi_{\tau}^{t, s}=\exp \left(-\frac{q\left(\frac{2}{2^{p}-2}\|t\|^{p}\right)}{\tau}\right)
$$

and letting $\beta=2^{1-p}$, we get the desired result.

Theorem 4.3 Let $(T, \xi, *, *)$ be an MB-algebra. Let $\varphi: T^{2} \rightarrow O+$ be a distribution function such that there exists a $\beta \in(0,1)$ with

$$
\varphi_{4 \beta \tau}^{t, s} \geq \varphi_{\tau}^{\frac{t}{2}, \frac{s}{2}}
$$


for all $t, s \in T$ and $\tau>0$. Suppose that random operators $G, H: \Gamma \times T \rightarrow T$ satisfy $G(\gamma, 0)=$ $H(\gamma, 0)=0$, (3.3) and (3.4). Then there are a unique $\mathbb{C}$-linear random operator $\Theta: \Gamma \times$ $T \rightarrow T$ and a unique stochastic derivation $\pi: \Gamma \times T \rightarrow T$ such that $[\Theta, \pi]: \Gamma \times T \rightarrow T$ is a stochastic derivation and

$$
\xi_{\tau}^{G(\gamma, t)-\Theta(\gamma, t)} * \xi_{\tau}^{H(\gamma, t)-\pi(\gamma, t)} \geq \varphi_{2(1-\beta) \tau}^{t, t}
$$

for all $t \in T, \gamma \in \Gamma$ and $\tau>0$.

Proof By Theorem 3.5, there exist a unique $\mathbb{C}$-linear random operator $\Theta: \Gamma \times T \rightarrow T$ and a unique stochastic derivation $\pi: \Gamma \times T \rightarrow T$ such that $[\Theta, \pi]: \Gamma \times T \rightarrow T$ is a stochastic a derivation.

Let $(S, \delta)$ be the generalized metric space defined in the proof of Theorem 4.1. Now, we consider the linear mapping $\Lambda: S \rightarrow S$ such that

$$
\Lambda(G, H)(\gamma, t):=\left(\frac{1}{2} G(\gamma, 2 t), \frac{1}{2} H(\gamma, 2 t)\right)
$$

for all $t \in T, \gamma \in \Gamma$. It follows from (4.3) that

$$
\begin{aligned}
\xi_{\tau}^{G(\gamma, t)-\frac{1}{2} G(\gamma, 2 t)} * \xi_{\tau}^{H(\gamma, t)-\frac{1}{2} H(\gamma, 2 t)} & \geq \varphi_{2 \tau}^{2 t, 2 t} \\
& \geq \varphi_{\frac{\tau}{2 \beta}}^{t, t}
\end{aligned}
$$

for all $t \in T, \gamma \in \Gamma$ and $\tau>0$. The proof will be finished by a similar method to the one used in the proofs of Theorems 3.3 and 4.1.

Corollary 4.4 Let $(T, \xi, *, *)$ be an MB-algebra. Assume that $q>0$ and $p<1$. Suppose that random operators $G, H: \Gamma \times T \rightarrow T$ satisfy $G(\gamma, 0)=H(\gamma, 0)=0$, (3.16) and (3.17). Then there are a unique $\mathbb{C}$-linear random operator $\Theta: \Gamma \times T \rightarrow T$ and a unique stochastic derivation $\pi: \Gamma \times T \rightarrow T$ such that $[\Theta, \pi]: \Gamma \times T \rightarrow T$ is a stochastic derivation and

$$
\xi_{\tau}^{G(\gamma, t)-\Theta(\gamma, t)} * \xi_{\tau}^{H(\gamma, t)-\pi(\gamma, t)} \geq \exp \left(-\frac{q\left(\frac{2}{22^{p}}\|t\|^{p}\right)}{\tau}\right)
$$

for all $t \in T, \gamma \in \Gamma$ and $\tau>0$.

Proof In Theorem 4.3, putting

$$
\varphi_{\tau}^{t, s}=\exp \left(-\frac{q\left(\frac{2}{2-2^{p}}\|t\|^{p}\right)}{\tau}\right)
$$

and letting $\beta=2^{p-1}$, we get the desired result.

\section{Acknowledgements}

The authors are thankful to the anonymous referees for giving valuable comments and suggestions which helped to improve the final version of this paper. 
Availability of data and materials

Not applicable.

\section{Competing interests}

The authors declare that they have no competing interests.

\section{Authors' contributions}

All authors conceived of the study, participated in its design and coordination, drafted the manuscript, participated in the sequence alignment, and read and approved the final manuscript.

\section{Author details}

'Department of Mathematics, Science and Research Branch, Islamic Azad University, Tehran, Iran. ${ }^{2}$ School of Mathematics, Iran University of Science and Technology, Tehran, Iran. ${ }^{3}$ Research Institute for Natural Sciences, Hanyang University, Seoul, Korea. ${ }^{4}$ Pedagogical Department of Mathematics \& Informatics, National and Kapodistrian University of Athens, Attikis, Greece.

\section{Publisher's Note}

Springer Nature remains neutral with regard to jurisdictional claims in published maps and institutional affiliations.

Received: 22 January 2020 Accepted: 4 May 2020 Published online: 13 May 2020

\section{References}

1. Schweizer, B., Sklar, A.: Probabilistic Metric Spaces. North-Holland Series in Probability and Applied Mathematics. North-Holland, New York (1983)

2. Šerstnev, A.N.: On the concept of a stochastic normalized space. Dokl. Akad. Nauk SSSR 149, 280-283 (1963) (in Russian)

3. Saadati, R:: Random Operator Theory. Elsevier/Academic Press, London (2016)

4. Hadzic, O., Pap, E. (eds.): Mathematics and Its Applications, vol. 536. Kluwer Academic, Dordrecht (2001)

5. Mirmostafaee, A.K.: Perturbation of generalized derivations in fuzzy Menger normed algebras. Fuzzy Sets Syst. 195, 109-117 (2012)

6. Saadati, R., Park, C.: Approximation of derivations and the superstability in random Banach $*$-algebras. Adv. Differ. Equ. 2018, Paper No. 418 (2018)

7. Naeem, R., Anwar, M.: Jessen type functionals and exponential convexity. J. Math. Comput. Sci. 17, 429-436 (2017)

8. Park, C., Yun, S.: Stability of cubic and quartic $\rho$-functional inequalities in fuzzy normed spaces. J. Nonlinear Sci. Appl. 9, 1693-1701 (2016)

9. Maleki, M.V., Vaezpour, S.M., Saadati, R.: Nonlinear stability of $\rho$-functional equations in latticetic random Banach lattice spaces. Mathematics 6(2), Paper No. 22 (2018)

10. Agarwal, R.P., Saadati, R., Salamati, A.: Approximation of the multiplicatives on random multi-normed space. J. Inequal. Appl. 2017, Paper No. 204 (2017)

11. Jang, S.Y., Saadati, R.: Approximation of an additive $\left(\varrho_{1}, \varrho_{2}\right)$-random operator inequality. J. Funct. Spaces 2020, Article ID 7540303 (2020)

12. Park, C., Eshaghi Gordji, M., Saadati, R.: Random homomorphisms and random derivations in random normed algebras via fixed point method. J. Inequal. Appl. 2012, Paper No. 194 (2012)

13. Rassias, J.M., Saadati, R., Sadeghi, G., Vahidi, J.: On nonlinear stability in various random normed spaces. J. Inequal. Appl. 2011, Paper No. 62 (2011)

14. Cho, Y.J., Rassias, T.M., Saadati, R.: Stability of Functional Equations in Random Normed Spaces, vol. 86. Springer, New York (2013)

15. Lu, G., Xin, J., Jin, Y., Park, C.: Approximation of general Pexider functional inequalities in fuzzy Banach spaces. J. Nonlinear Sci. Appl. 12, 206-216 (2019)

16. El-Moneam, M.A., Ibrahim, T.F., Elamody, S.: Stability of a fractional difference equation of high order. J. Nonlinear Sci. Appl. 12, 65-74 (2019)

17. Keltouma, B., Elhoucien, E., Rassias, T.M. Ahmed, R.: Superstability of Kannappan's and Van Vleck's functional equations. J. Nonlinear Sci. Appl. 11, 894-915 (2018)

18. Ding, Y.: Ulam-Hyers stability of fractional impulsive differential equations. J. Nonlinear Sci. Appl. 11, 953-959 (2018)

19. Binzar, T., Pater, F., Nadaban, S.: On fuzzy normed algebras. J. Nonlinear Sci. Appl. 9, 5488-5496 (2016)

20. Hadžić, O.: A random fixed point theorem for multivalued mappings of Ćirić's type. Mat. Vesn. 3(16)(31)(4), 397-401 (1979)

21. Todorčević, V.: Harmonic Quasiconformal Mappings and Hyperbolic Type Metrics. Springer, Cham (2019)

22. Patle, P., Patel, D., Aydi, H., Radenović, S.: On $\mathcal{H}^{+}$type multivalued contraction and its applications in symmetric and probabilistic spaces. Mathematics 7(2), Paper No. 144 (2019)

23. Ndolane, S.: Exponential form for Lyapunov function and stability analysis of the fractional differential equations. J. Math. Comput. Sci. 18, 388-397 (2018)

24. Wu, R., Li, L.: Note on the stability property of the boundary equilibrium of a May cooperative system with strong and weak cooperative partners. J. Math. Comput. Sci. 20, 58-63 (2020)

25. Lee, Y., Jung, S.: A fixed point approach to the stability of a general quartic functional equation. J. Math. Comput. Sci. $20,207-215(2020)$

26. Piri, H., Rahrovi, S., Kumam, P.: Generalization of Khan fixed point theorem. J. Math. Comput. Sci. 17, 76-83 (2017)

27. Shoaib, A., Azam, A., Arshad, M., Ameer, E.: Fixed point results for multivalued mapping on sequence in a closed ball with applications. J. Math. Comput. Sci. 17, 308-316 (2017)

28. Brzdek, J., Ciepliński, K.: A fixed point theorem in n-Banach spaces and Ulam stability. J. Math. Anal. Appl. 470 632-646 (2019) 
29. Park, C.: Lie bracket derivation-derivations in complex Banach algebras. Preprint

30. Nădăban, S., Bînzar, T., Pater, F.: Some fixed point theorems for $\varphi$-contractive mappings in fuzzy normed linear spaces J. Nonlinear Sci. Appl. 10(11), 5668-5676 (2017)

31. Diaz, J.B., Margolis, B.: A fixed point theorem of the alternative, for contractions on a generalized complete metric space. Bull. Am. Math. Soc. 74, 305-309 (1968)

32. Cădariu, L., Radu, V.: Fixed points and the stability of Jensen's functional equation. J. Inequal. Pure Appl. Math. 4(1), Article 4 (2003)

33. Cădariu, L., Radu, V.: Fixed points and the stability of quadratic functional equations. An. Univ. Vest. Timiş., Ser Mat.-Inform. 41, 25-48 (2003)

34. Park, C.: Homomorphisms between Poisson JC*-algebras. Bull. Braz. Math. Soc. 36, 79-97 (2005)

35. Miheț, D., Radu, V.: On the stability of the additive Cauchy functional equation in random normed spaces. J. Math Anal. Appl. 343, 567-572 (2008)

36. Miheț, D., Saadati, R.: On the stability of the additive Cauchy functional equation in random normed spaces. Appl. Math. Lett. 24, 2005-2009 (2011)

\section{Submit your manuscript to a SpringerOpen ${ }^{\circ}$} journal and benefit from:

- Convenient online submission

Rigorous peer review

- Open access: articles freely available online

- High visibility within the field

- Retaining the copyright to your article

Submit your next manuscript at $\gg$ springeropen.com 